\title{
Src activation by $\beta$-adrenoreceptors is a key switch for tumour metastasis
}

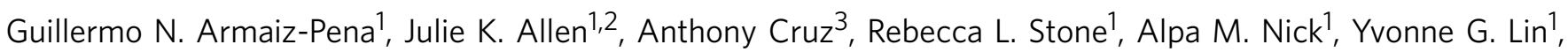
Liz Y. Han', Lingegowda S. Mangala1,4, Gabriel J. Villares ${ }^{2,5}$, Pablo Vivas-Mejia6,7, Cristian Rodriguez-Aguayo6, Archana S. Nagaraja 1,2, Kshipra M. Gharpure ${ }^{1,2}$, Zheng Wu ${ }^{8,9}$, Robert D. English ${ }^{8}$, Kizhake V. Soman ${ }^{8,9}$, Mian M.K. Shahzad ${ }^{1}$, Maya Zigler ${ }^{2,5}$, Michael T. Deavers ${ }^{10}$, Alexander Zien ${ }^{11}$, Theodoros G. Soldatos ${ }^{11}$, David B. Jackson ${ }^{11}$, John E. Wiktorowicz ${ }^{8,9}$, Madeline Torres-Lugo ${ }^{12}$, Tom Young ${ }^{13}$, Koen De Geest ${ }^{14}$, Gary E. Gallick ${ }^{15}$, Menashe Bar-Eli ${ }^{5}$, Gabriel Lopez-Berestein ${ }^{4,5}, 6$, Steve W. Cole ${ }^{16}$, Gustavo E. Lopez ${ }^{3}$, Susan K. Lutgendorf ${ }^{14,17} \&$ Anil K. Sood ${ }^{1,4,5}$

Noradrenaline can modulate multiple cellular functions important for cancer progression; however, how this single extracellular signal regulates such a broad array of cellular processes is unknown. Here we identify Src as a key regulator of phosphoproteomic signalling networks activated in response to beta-adrenergic signalling in cancer cells. These results also identify a new mechanism of Src phosphorylation that mediates beta-adrenergic/PKA regulation of downstream networks, thereby enhancing tumour cell migration, invasion and growth. In human ovarian cancer samples, high tumoural noradrenaline levels were correlated with high $\mathrm{pSrc} \mathrm{Y}^{419}$ levels. Moreover, among cancer patients, the use of beta blockers was significantly associated with reduced cancer-related mortality. Collectively, these data provide a pivotal molecular target for disrupting neural signalling in the tumour microenvironment.

\footnotetext{
${ }^{1}$ Department of Gynecologic Oncology and Reproductive Medicine, The University of Texas MD Anderson Cancer Center, Houston, Texas 77030, USA.

${ }^{2}$ Cancer Biology Program, Graduate School of Biomedical Sciences, The University of Texas Health Science Center, Houston, Texas 77030, USA.

${ }^{3}$ Department of Chemistry, University of Puerto Rico, Mayaguez, Puerto Rico 00681, USA. ${ }^{4}$ Center for RNA Interference and Non-coding RNA, The University of Texas MD Anderson Cancer Center, Houston, Texas 77030, USA. ${ }^{5}$ Department of Cancer Biology, The University of Texas MD Anderson Cancer Center, Houston, Texas 77030, USA. ${ }^{6}$ Department of Experimental Therapeutics, The University of Texas MD Anderson Cancer Center, Houston, Texas 77030, USA. ${ }^{7}$ Department of Biochemistry and Cancer Center, Medical Sciences Campus, University of Puerto Rico, San Juan, Puerto Rico 00935, USA. ${ }^{8}$ Biomolecular Resource Facility, The University of Texas Medical Branch, Galveston, Texas 77555, USA. ${ }^{9}$ Department of Biochemistry and Molecular Biology, The University of Texas Medical Branch, Galveston, Texas 77555, USA. ${ }^{10}$ Department of Pathology, The University of Texas MD Anderson Cancer Center, Houston, Texas 77030, USA. ${ }^{11}$ Molecular Health GmbH, Belfortstr. 2, 69115 Heidelberg, Germany. ${ }^{12}$ Department of Chemical Engineering, University of Puerto Rico, Mayaguez, Puerto Rico 00681, USA. ${ }^{13}$ Department of Chemistry, Lehman College, Bronx, New York 10468, USA. ${ }^{14}$ Division of Gynecologic Oncology, Department of Obstetrics and Gynecology, University of lowa, lowa City, lowa 52242, USA. ${ }^{15}$ Department of Genitourinary Medical Oncology, The University of Texas MD Anderson Cancer Center, Houston, Texas 77030, USA. ${ }^{16}$ Division of Hematology-Oncology, Department of Medicine, University of California, Los Angeles, California 90095, USA. ${ }^{17}$ Department of Psychology and Urology, and Holden Comprehensive Cancer Center, University of lowa, lowa City, lowa 52242, USA. Correspondence and requests for materials should be addressed to A.K.S. (email: asood@mdanderson.org).
} 
T he tumour microenvironment is an important determinant of cancer progression, and microenvironmental distribution of neural and endocrine signals has now been identified as a key mediator of these dynamics ${ }^{1,2}$. For example, signalling resulting from activation of beta-adrenergic receptors (ADRB) by noradrenaline (NA) affects a broad array of processes involved in the progression of various cancer types ${ }^{1,2}$. However, the diversity of intracellular signalling pathways and cellular processes modulated by this single extracellular signal is surprising. For example, our previous work determined that sympathetic nervous system activity can directly enhance the pathogenesis of ovarian carcinoma by protecting tumour cells from anoikis ${ }^{3}$, promoting tumour cell invasion ${ }^{4-6}$ and tumourassociated angiogenesis ${ }^{2,7}$. We found that these effects were mediated through activation of tumour cell ADRB2, but its downstream signalling pathways are not well understood.

Here, we sought to determine key regulators of the cellular phosphoproteome following NA stimulation of ADRB in cancer cells. We demonstrate that ADRB signalling leads to Src activation by a unique PKA-mediated mechanism, which is critical to the regulation of phosphoproteomic networks associated with ovarian cancer progression.

\section{Results}

NA activated signalling networks. Following treatment of SKOV3ip1 cells with NA, proteins from treated and untreated cells were separated by 2D gel electrophoresis and stained for total and phosphorylated proteins (Supplementary Fig. S1a,b). Quantitative analyses of these samples, followed by mass spectrometry analysis identified 24 proteins with altered expression levels and 39 with differential phosphorylation (Supplementary Tables S1 and 2 and Supplementary Data 1 and 2). For each of these proteins, we identified kinases that may be upstream by up to two levels (the kinase targets another kinase, which targets the protein). To identify putative key mediators, all involved kinases were scored by the number of identified downstream proteins. The highest score was achieved for Src (Fig. 1 and Supplementary Fig. S2a). To validate this finding, lysates from NA-treated tumour cells were subjected to immunoblotting, which confirmed the results obtained in our analysis (Supplementary Fig. S2b). Additionally, treatment with either dasatinib or Src small interfering RNA (siRNA) abrogated NA-induced changes (Supplementary Fig. S2b). Next, we sought to determine the functional and biological roles of Src in promoting tumour growth in response to increased adrenergic signalling.

Beta-adrenergic receptors mediate NA-induced Src activation. We first examined Y419 phosphorylation following NA stimulation. As ovarian cancer cells do not produce NA (data not shown), we exposed cells to various NA concentrations known to be present in ovarian tissues and tumours under physiological and stress conditions ${ }^{2,8}$. In the ADRB-positive HeyA8 and SKOV3ip1 human ovarian cancer cells ${ }^{2,9}, \mathrm{pSrc}^{\mathrm{Y} 419}$ levels increased markedly (at least three-fold) following exposure to $100 \mathrm{nM}-10 \mu \mathrm{M}$ NA (Fig. 2a and Supplementary Fig. S3a). These increases are comparable to those seen by growth factor-mediated Src phosphorylation, as observed in Supplementary Fig. S3b. To show that Y419 phosphorylation leads to Src activation, we performed a kinase assay where focal adhesion kinase (FAK) was exposed to Src or a combination of Src with AP23846. Upon interaction with Src, $\mathrm{FAK}^{\mathrm{Y} 397}$ phosphorylation was substantially increased, while AP23846 blocked this effect (Supplementary Fig. S3c). Additionally, we show that FAK exposure to Src results in increased phosphorylation at Y925 that is not seen in the absence of ATP (Supplementary Fig. S3c). Similar responses to
NA with regard to Y419 phosphorylation were noted with ADRBpositive breast cancer and melanoma cell lines (Supplementary Fig. S3d). In contrast, NA stimulation of the ADRB-deficient A2780-PAR cells ${ }^{2}$ or hydrocortisone treatment of SKOV3ip1 cells did not increase pSrc ${ }^{\mathrm{Y} 19}$ levels (Supplementary Fig. S3e and data not shown). Propranolol blocked NA-mediated Src activation (Supplementary Fig. S3e). Given the known role of $\mathrm{pSrc}^{\mathrm{Y} 530}$ dephosphorylation in Src activation, we also probed for $\mathrm{pSrc}^{\mathrm{Y} 530}$ following NA stimulation. There was no change in $\mathrm{pSrc}^{\mathrm{Y} 530}$ phosphorylation, suggesting that the NA-induced Src activation was solely mediated by phosphorylation at Y419 (Supplementary Fig. S3f,g). To further corroborate these findings, HeyA8 cells were treated with isoproterenol $(10 \mu \mathrm{M})$, which resulted in $\mathrm{Src}^{\mathrm{Y} 419}$ phosphorylation within 5 min (Supplementary Fig. S3h). Butoxamine, blocked the NA-induced Src activation (Fig. 2b). To examine the specificity of $\mathrm{ADRB}$ receptors in mediating NAinduced activation of Src, we utilized ADRB1- or ADRB2targeted siRNA capable of reducing levels of each protein by $>80 \%$ (Supplementary Fig. S3i). Similar to the effects with inhibitors, ADRB1 and ADRB2 siRNA abrogated NA-induced Src activation (Fig. 2c). Next, we created stable clones of A2780PAR cells transfected with an ADRB2 construct. After confirming ADRB2 expression (Supplementary Fig. S3j), we treated these cells with NA, which resulted in increased $\mathrm{Src}^{\mathrm{Y} 419}$ phosphorylation (Supplementary Fig. S3k).

NA-induced Src activation is mediated by cAMP/PKA. We next performed a series of experiments to delineate the signalling pathway involved in NA-mediated Src activation. Treatment of SKOV3ip1 cells with forskolin resulted in rapid Src ${ }^{\mathrm{Y} 419}$ phosphorylation (Supplementary Fig. S3l). To test whether PKA was involved in NA-mediated Src activation, cells were treated with dibutyryl-cAMP (dbcAMP), resulting in rapid Src ${ }^{\mathrm{Y} 419}$ phosphorylation (Fig. 2d). Furthermore, PKA silencing by siRNA or PKA inhibitors prevented NA-mediated Src activation (Fig. 2e and Supplementary Fig. S3m,n). Immunofluorescence analyses verified that upon NA stimulation, Src localizes to the focal adhesions in SKOV3ip1 cells (Fig. 2f,g and Supplementary Fig. S3o).

$\mathrm{pSrc}^{\mathrm{S} 17}$ is required for NA-induced Src activation. As PKA is a serine-threonine kinase, the paradoxical increase in Src tyrosine phosphorylation prompted us to consider potential underlying mechanisms. Src contains a single consensus PKA site at residues surrounding S17 (Supplementary Fig. S4a) ${ }^{10}$. To test whether NA and dbcAMP-mediated induction of $\mathrm{Src}^{\mathrm{S} 17}$ phosphorylation was PKA-dependent, we treated HeyA8 cells with NA or dbcAMP. Both treatments rapidly increased $\mathrm{pSrc}^{\mathrm{S} 17}$ levels (Fig. 3a and Supplementary Fig. S4b). Furthermore, in ADRB2-null A2780PAR cells stably transfected with ADRB2, NA stimulation rapidly increased PKA activity, $\mathrm{Src}^{\mathrm{S} 17}$ phosphorylation and Src activation (Supplementary Fig. S4c,d). To determine whether Ser17 phosphorylation is a prerequisite for NA-induced $\mathrm{Src}^{\mathrm{Y} 419}$ phosphorylation, mouse embryonic fibroblast cells null for Src, Yes or Fyn (SYF) were transfected with plasmids containing either wild-type (WT) Src or Src mutated at S17 (S17A). To verify that NA could increase PKA activity in SYF cells, we measured phospho-PKA substrate levels in these cells. As expected, NA rapidly increased the levels of phospho-PKA substrates (Supplementary Fig. S3e). In addition, after verifying the transfection efficiency and confirming that WT and S17A Src were transiently expressed at similar levels (Supplementary Fig. $\mathrm{S} 4 \mathrm{f}, \mathrm{g})$, we exposed them to NA or dbcAMP. Src ${ }^{419}$ and $\mathrm{Src}^{\mathrm{SP}} \mathrm{7}$ were rapidly phosphorylated in WT Src-expressing cells following 


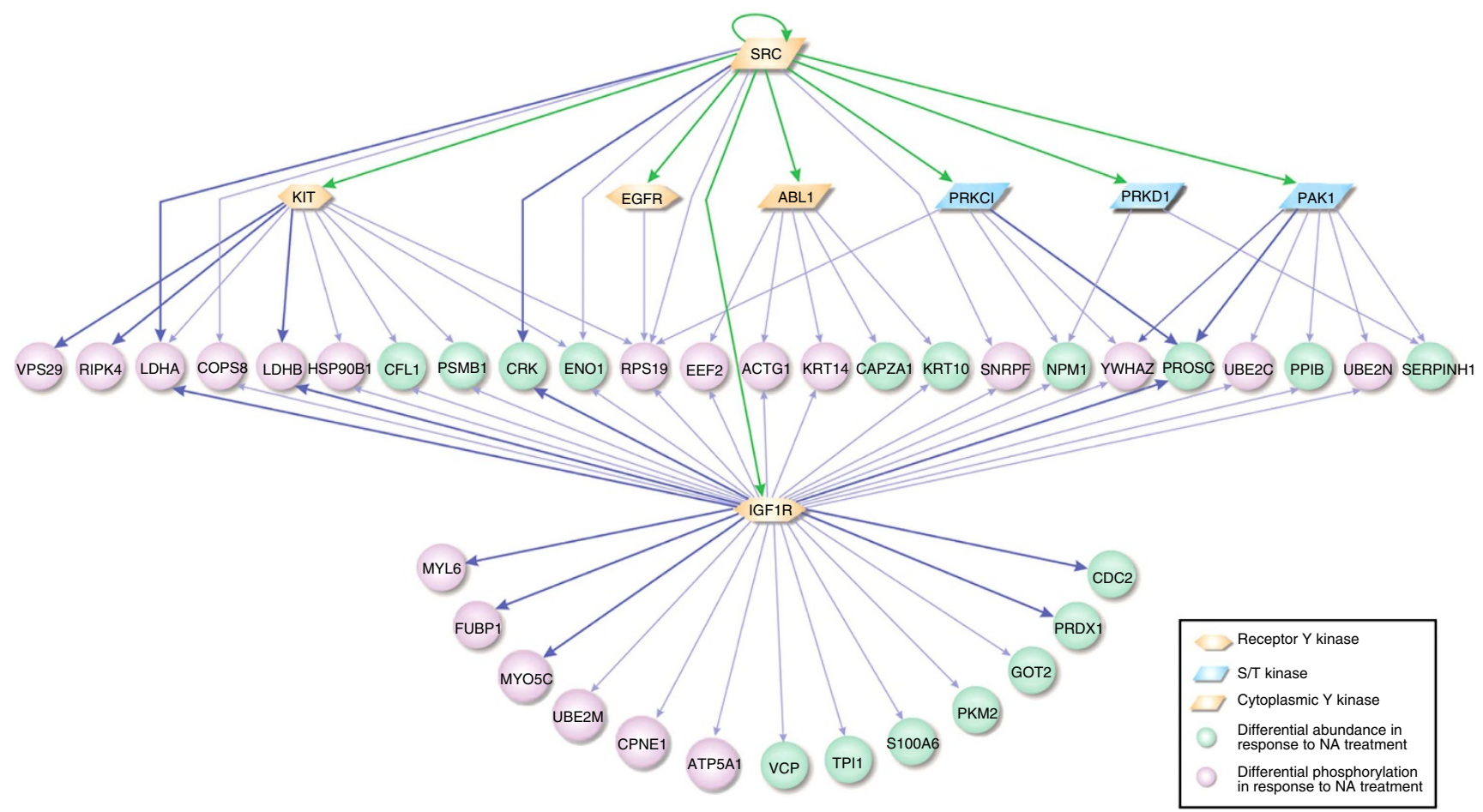

Figure 1 | Putative phosphorylation cascade triggered by the NA-induced activation of Src. In this predicted network (see 'Signaling Network Analysis' in 'Methods'), the components connecting Src to the NA-responsive proteins are shown as arrows: green, experimentally observed phosphorylation according to PhosphoELM; dark blue, predicted by NetworKIN; light blue, predicted by NetworKIN for a close homologue of the target.

NA treatment, but not in the S17A Src-transfected cells (Fig. 3b,c and Supplementary Fig. S4h).

Interaction between pS17 and Src exposes Y419. The contribution of the Src N-terminus, where S17 resides, and specifically its unique domain to Src activation is not known, as the reported Src crystal structure does not include the first 82 aminoacid residues ${ }^{11}$. To understand how S17 phosphorylation might lead to subsequent Y419 phosphorylation, we performed molecular dynamic simulations. First, we obtained the atomic coordinates of c-Src from the Protein Data Bank (Code:2SRC) ${ }^{12}$, and proceeded to eliminate phospho-aminophosphonic acidadenylate ester (ANP) and water molecules, leaving Src in its inactive form (Fig. 4a). Subsequently, we ran preliminary simulations with a computer designed full-length $\mathrm{N}$-terminal attached to the known Src crystal structure. After performing extensive annealing molecular dynamic simulations, no significant data in the time scale of the simulation could be obtained (data not shown). Next, we constructed a model peptide that resembled the N-terminus where Ser17 resides (K9-E19 fragment). An initial estimate of the secondary structure was generated using the PSIPRED server and submitted to the AbinitioRelax application as implemented in Rosetta 3.0 (ref. 13). This application resulted in 5,000 possible structures and only conformations in structural agreement with the initial estimate were used in the second stage of this procedure. We chose the structure that permitted the accommodation of the phosphate group in the S17 position without disturbing the structure of the peptide (Supplementary Fig. S5a,b).

To identify possible cavities in the structure of the protein where the peptide could interact, we subjected the inactive structure of c-Src to a Computed Atlas of Surface Topography of Proteins $^{14}$ analysis using the University of California at San
Francisco Chimera interface. The obtained results suggested three possible cavities with an area and volume large enough to accommodate the designed peptide (Fig. $4 \mathrm{~b}$ and Supplementary Fig. S5c). One of these cavities is the active site where ANP binds, which is not spatially accessible for the peptide until the protein is in its active conformation. To elucidate which of the cavities was the best candidate, the structure of Src was compared with the human tyrosine kinase c-Abl, (PDB code: $2 \mathrm{fo} 0$ ). Both of these structures were aligned using the Multiseq plug-in ${ }^{15}$ in the Visual Molecular Dynamics package (VMD 1.8.4) ${ }^{16}$.c-Abl has a different auto-inactivation mechanism compared with c-Src. However, this mechanism involves interaction of the N-terminal Myristoyl group with the four major helices (H11, H16, H18 and H20) in the kinase domain. This structure includes residues $65-82$ that are part of the N-terminal cap of c-Abl. Owing to the structural resemblance between $\mathrm{Src}$ and c-Abl (47\% sequence homology) ${ }^{17}$, it is plausible that the conformation of the N-terminus in c-Abl is similar to the $\mathrm{N}$-terminus in Src, and hence it is possible to conclude that the $\mathrm{N}$-terminus of $\mathrm{c}$-Src follows a similar trajectory as the N-terminus of c-Abl, a trajectory that is well known. On the basis of this analysis, Src has only one possible cavity where the peptide can be inserted. This cavity is located near the C-terminal, and between the SH2 and kinase domain (Fig. 4b and Supplementary Fig. S5c). This cavity is also the most accessible of the three cavities we identified. Furthermore, we used molecular dynamics to simulate a hydrated Src and performed solvation analyses $^{18-20}$ of the selected cavity to ensure that water displacement from the allosteric site is thermodynamically favourable. This approach identified eight high solvent density regions in the cavity that are thermodynamically unfavourable and displaced after the peptide binds. Water displacement from these regions to more thermodynamically favourable bulk biological fluid strongly suggests that the peptide can bind at this site (Supplementary Fig. S5d). 
a

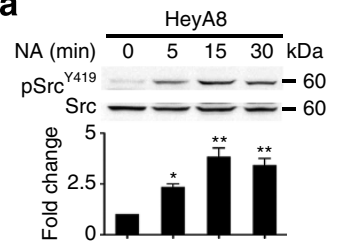

C

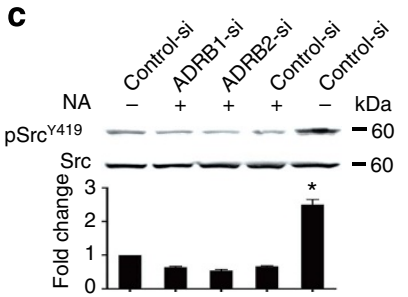

$\mathbf{f}$
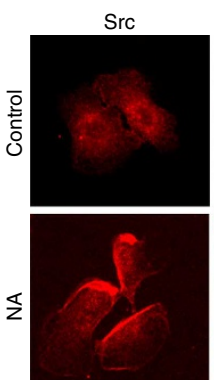

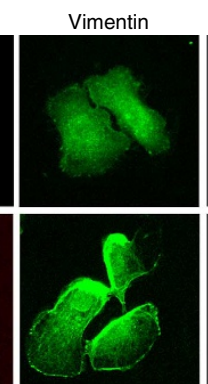

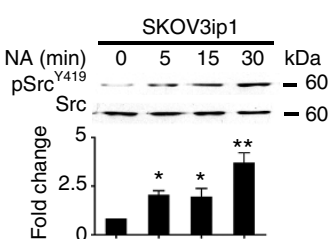

d
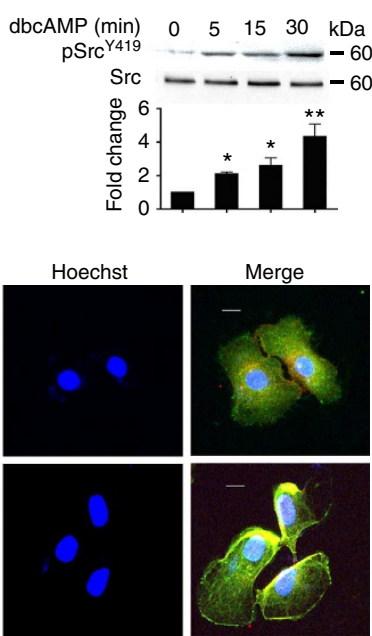

b

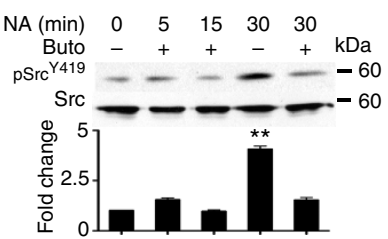

e

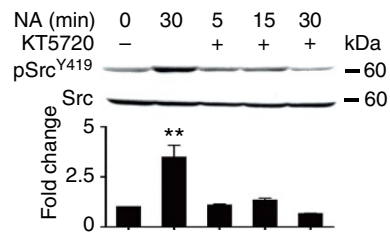

g

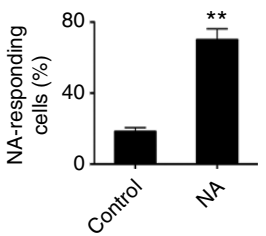

Figure 2 | NA-induced Src activation is mediated by an ADRB/cAMP/PKA mechanism. (a) Effect of $10 \mu \mathrm{M}$ NA on Src ${ }^{Y 419}$ phosphorylation in HeyA8 and SKOV3ip1 cells. ${ }^{\star} P<0.01,{ }^{\star \star} P<0.001$; two-tail Student's $t$-test. (b) HeyA8 cells were incubated for $1 \mathrm{~h}$ with $1 \mu \mathrm{M}$ butoxamine (Buto, $\beta_{2}$ antagonist), exposed to $10 \mu \mathrm{M} N A$ and probed for $\mathrm{pSrc}{ }^{\mathrm{Y} 419}{ }^{\star}{ }^{\star} P<0.001$; two-tail Student's $t$-test. (c) Similar experiments were performed with siRNA targeted against ADRB1 (ADRB1 si) or ADRB2 (ADRB2 si) in SKOV3ip1 cells. ${ }^{\star} P<0.001$; two-tail Student's t-test. (d) Effect of $50 \mu M$ dbcAMP (PKA agonist) on pSrc ${ }^{4} 19$ in SKOV3ip1 cells. ${ }^{\star} P<0.01,{ }^{\star \star} P<0.001$; two-tail Student's $t$-test. (e) HeyA8 cells were exposed to $10 \mu \mathrm{M} \mathrm{KT5720}$ (PKA antagonist) for $1 \mathrm{~h}$, stimulated with $10 \mu \mathrm{M} N A$ and probed for $\mathrm{pSrc}{ }^{\mathrm{Y} 419}$. ${ }^{\star} P<0.001$; two-tail Student's $t$-test. (f) SKOV3ip1 cells were treated with $10 \mu \mathrm{M} N \mathrm{NA}$, and Src was visualized by immunofluorescence (scale bar, $9.375 \mu \mathrm{m}$ ). (g) Quantification of cellular response to NA (measured as cells with increased Src expression at the focal adhesions) is shown in graphs ${ }^{*} P<001$; two-tail Student's $t$-test. In panels (a-e), the immunoblot is shown at the top and quantification of pSrc band intensity relative to the intensity of the total Src band is shown below.

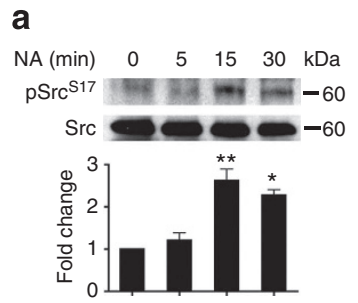

C

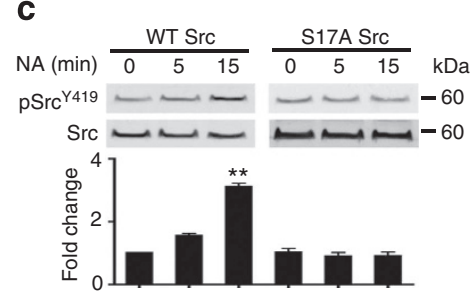

b
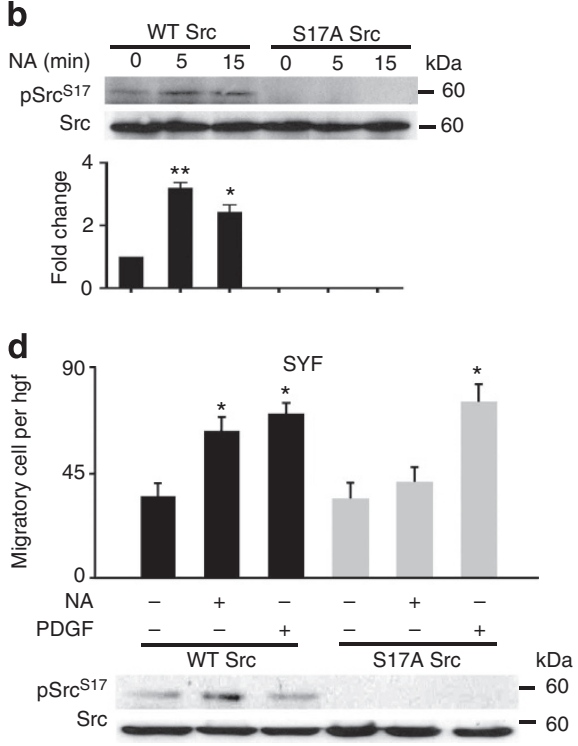

Figure 3 | NA-induced Src ${ }^{\mathbf{S 1 7}}$ phosphorylation on SYF cells is required for Src activation. (a) HeyA8 cells were treated with $10 \mu \mathrm{M}$ NA and probed for $\mathrm{pSrc}{ }^{517}$. ${ }^{\star} P<0.01 ;{ }^{\star \star} P<0.001$; two-tail Student's $t$-test. SYF-null cells transfected with either WT or mutant Src (S17A) were stimulated with $10 \mu M$ NA and immunoblotted for $(\mathbf{b}) \mathrm{pSrc}{ }^{S 17}$ or (c) $\mathrm{pSrc}{ }^{Y 419}$. ${ }^{\star} P<0.01$; ${ }^{\star \star} P<0.001$; two-tail Student's $t$-test. (d) SYF-null cells transfected with either WT or mutant Src (S17A) were stimulated with $10 \mu \mathrm{M} \mathrm{NA}$ or PDGF $\left(20 \mathrm{ng} \mathrm{ml}^{-1}\right)$ and subjected to a migration assay or western blot analysis for pSrc ${ }^{S 17}$ expression. ${ }^{\star} P<0.01$; two-tail Student's $t$-test. In panels $(\mathbf{a}-\mathbf{c})$, the immunoblot is shown at the top and quantification of pSrc band intensity relative to the intensity of the total Src band is shown below. 
a

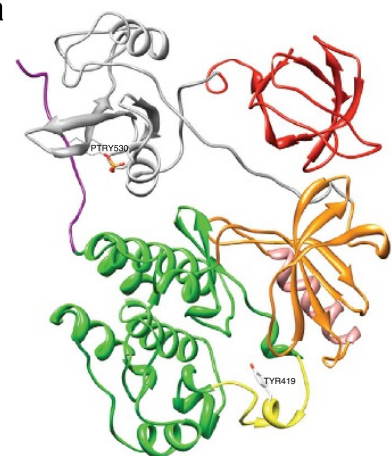

C

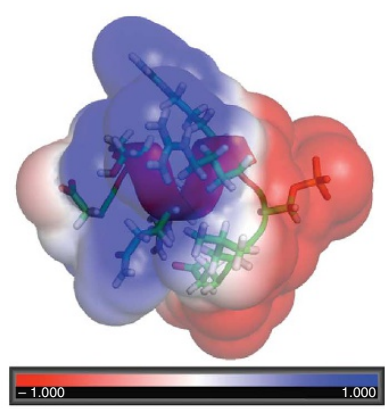

f

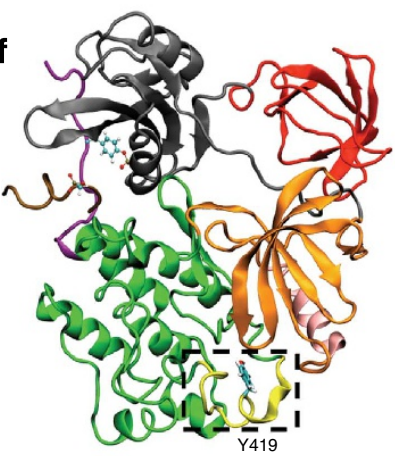

g

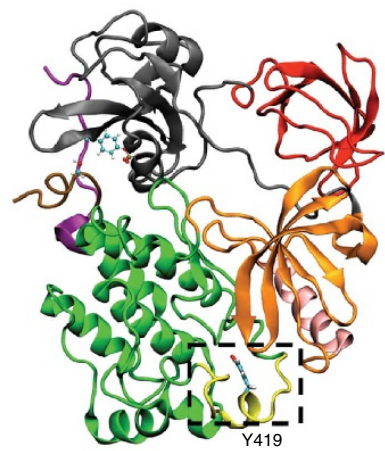

b

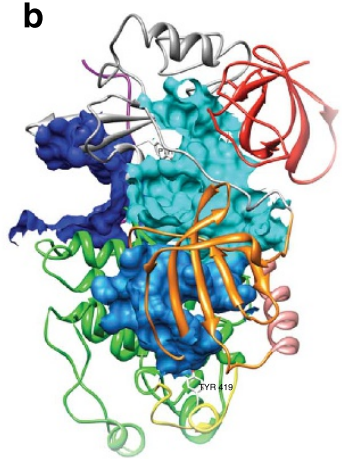

d
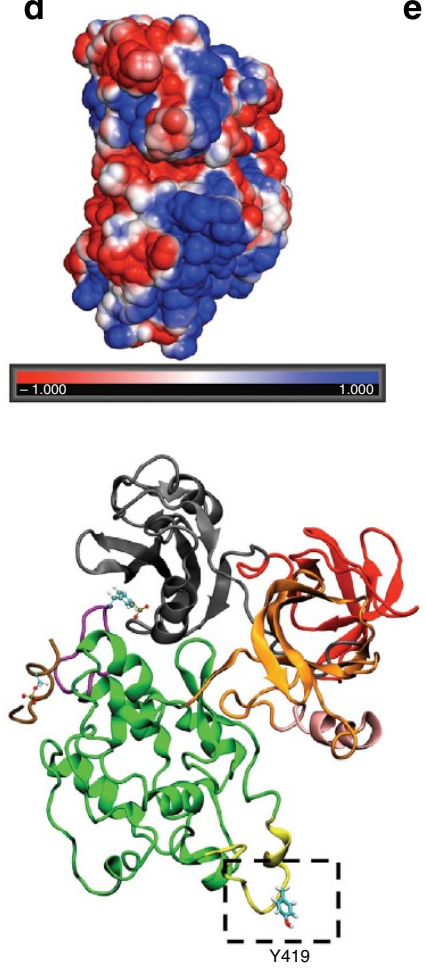

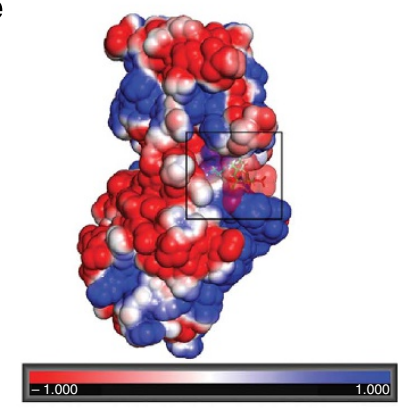

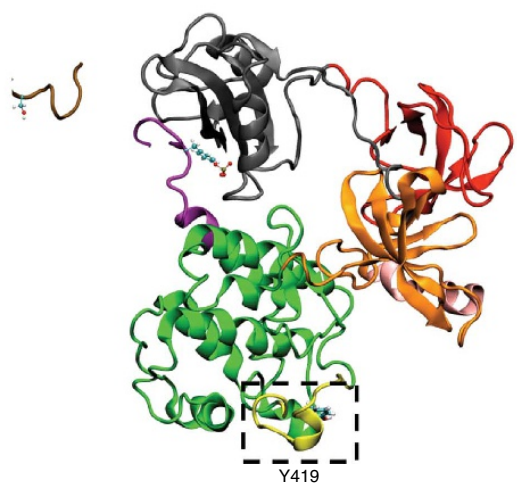

Figure 4 | Interaction between pS17 and Src results in conformational changes that expose Y419. (a) Inactive form of the protein obtained from the protein data bank (PDB code: 2SRC). The ANP and all the water molecules were deleted from the PDB structure. (b) View of the three identified cavities where the peptide could be inserted. Charge distribution analysis of (c) designed model peptide, (d) Src and (e) the model peptide docked in the proposed cavity. The red and blue regions represent negative and positive charges, respectively. (f) After the phosphopeptide was exposed to Src for 36 ns, the kinase domain moved, completely exposing Y419. (g) When Src was exposed to an unphosphorylated peptide, no significant movement was observed and Y419 was not exposed. The SH3, SH2, N-lobe and alphaC domains are shown in red, grey, orange and pink, respectively. The C-lobe, A-loop, C-terminus and phosphopeptide are shown in green, yellow, purple and brown, respectively.

As the peptide contains a high content of charged residues (63.6\%), a charge distribution analysis was performed using the Adaptive Poisson-Boltzmann Solver within $\mathrm{Pymol}^{21}$, while the charges and radii used were obtained from the amber99SB force field ${ }^{22}$. Our results demonstrated that the chosen cavity had a highly negative charged surface in the interior that could 
accommodate the positive portion of the peptide while exhibiting a positively charged entrance that could accommodate the negative charge on the other part of the peptide (Fig. 4c-e). Hence, from an electrostatic point of view, this cavity provides the correct environment for protein-peptide interaction.

Finally, we performed molecular dynamic simulations using the inactive structure of c-Src with the phosphorylated peptide docked to the cavity that we identified in our previous simulations. Our results demonstrate that the Src/phosphorylated peptide model undergoes significant structural changes in the kinase domain, that is, exposure of the Y419 residue without alteration of the C-terminus (Fig. 4f, Supplementary Movie 1). Additionally, our simulation showed that the $\mathrm{SH} 2$ domain maintained its closed conformation. These two changes are characteristic of the activated form of Src. Supplementary Fig. S5e depicts a probability contour of the contacts between the phosphorylated peptide and the protein throughout the simulated timeframe. In contrast, a peptide containing a S17A mutation had a negligible difference in its polarity when compared to an unphosphorylated peptide and due to size of this system, we assumed this difference to be insignificant. Hence, we performed simulations of Src in the presence of a peptide lacking S17 phosphorylation. Furthermore, no significant alteration of the protein structure was observed when the simulation was run with this peptide (Fig. $4 \mathrm{~g}$ and Supplementary Movie 2). Moreover, during the simulated time, the unphosphorylated peptide leaves the cavity, suggesting that the interaction between the peptide and Src is not as strong as with the phosphorylated peptide. To confirm these results, we performed a kinase assay where Src was exposed to the same phosphorylated peptide used in our molecular dynamic simulations. Upon interaction with the phosphorylated peptide, $\mathrm{Src}^{\mathrm{Y} 419}$ phosphorylation was substantially elevated, resulting in enhanced enzymatic activity and increased Src-dependent FAK phosphorylation at Y861 (Supplementary Fig. S5f-h).

Src mediates NA-induced cell migration and invasion. To determine the functional effects of NA-induced $\mathrm{Src}^{\mathrm{S} 17}$ phosphorylation, we first examined its effects on cell migration. In WT Src-transfected SYF cells, NA increased S17 phosphorylation, while PDGF treatment did not (Fig. 3d). Furthermore, NA and PDGF treatment significantly increased migration of WT Srctransfected SYF cells $(P<0.01)$. In contrast, NA did not stimulate the migration of S17A Src-transfected SYF cells, while PDGF still promoted cell migration $(P<0.01$; Fig. $3 \mathrm{~d})$. In non-transfected SYF cells, NA failed to induce an increase in the migratory ability of these cells (Supplementary Fig. S4i). These data indicate that NA-induced Src activation requires direct phosphorylation of S17, which results in Src ${ }^{Y} 419$ phosphorylation, a mechanism distinct from Src activation by classic growth factor/growth factor receptor interactions.

To determine whether Src or PKA were responsible for mediating the stimulatory effects of catecholamines, we used three different Src siRNA sequences that silenced Src expression by $>80 \%$, AP23846 or KT5720 (Supplementary Fig. S6a and data not shown). NA treatment significantly increased the invasive potential of SKOV3ip1 and HeyA8 cells $(P<0.01$; Fig. 5a and Supplementary Fig. S6c,d). Src siRNA or AP23846 completely abrogated the NA-induced increase in invasion in both cell lines (Fig. 5a and Supplementary Fig. S6c), while KT5720 blocked NAinduced invasion in SKOV3ip1 cells (Supplementary Fig. S6d). Furthermore, NA-induced migration of SKOV3ip1 or HeyA8 cells was abrogated with Src siRNA treatment or AP23846 (Fig. 5b and Supplementary Fig. S6b). Next, we used the poorly invasive A2780-ADRB2 cells because they express very low levels of Src when compared with a panel of ovarian cancer cells (data not shown). After transiently transfecting these cells with a vector carrying either WT Src or S17A Src, invasion assays were carried out. WT Src potentiated the effect of NA on the invasiveness of these cells, while the introduction of S17A Src failed to have a similar effect when compared with non-transfected A2780ADRB2 cells (Supplementary Fig. S6e). Additionally, we transfected ID8 ${ }^{\text {VEGF }}$ murine ovarian carcinoma cells with human WT Src or S17A Src and then silenced endogenous Src by treating them with siRNA targeted against murine Src. Cells were then exposed to NA and subjected to migration and invasion assays. NA treatment resulted in increased cancer cell migration and invasion in WT Src-ID8 ${ }^{\text {VEGF }}$ cells, but not in the S17A SrcID8 ${ }^{\text {VEGF }}$ cells (Supplementary Fig. S6f,g).

We next asked if an increase in Src activity, upon adrenergic stimulation, could result in the induction of genes known to be mediators of cell motility and invasion. To address this question, we performed a cDNA microarray analysis of NA-treated SKOV3ip1 cells and identified genes relevant for tumour cell invasion. Our analysis revealed a significant increase in several such genes following NA treatment, and this increase was blocked by Src silencing (Supplementary Fig. S6h).

Restraint stress-induced tumour growth is mediated by Src. To test the biological significance of adrenergic-mediated Src activation, we utilized an in vivo restraint stress model $^{2}$. In this model, tumours from animals exposed to daily restraint had substantially increased levels of pSrc ${ }^{\mathrm{Y} 419}$ (Supplementary Fig. S7b) compared with controls. Src siRNA was incorporated into 1,2-dioleoyl-sn-glycero-3-phosphatidylcholine (DOPC) nanoliposomes for in vivo delivery. After confirming $>80 \%$ reduction in Src levels in vivo (Supplementary Fig. S7a), we treated control or restrained mice ( $n=10$ per group) bearing SKOV3ip1 or HeyA8 tumours with control siRNA-DOPC or Src siRNA-DOPC. As expected, daily restraint significantly increased tumour growth (Fig. 5c,d). This increase was completely blocked by Src siRNA-DOPC (Fig. 5c,d). Furthermore, the number of tumour nodules was also reduced by Src siRNA-DOPC in both the HeyA8 and SKOV3ip1 models (Fig. 5c,d). These results were confirmed by additional experiments that utilized two different Src-specific siRNA sequences and the Src small molecule inhibitor, AP23846 (Supplementary Fig. S7c,d). Next, we analysed tumour tissues from daily restraint versus control mice that were treated with control siRNA or Src-specific siRNA with the proliferation markers phospho-histone h3 and proliferating cell nuclear antigen and the apoptotic marker cleaved caspase 3. Restraint stress resulted in increased cell proliferation that was abrogated by Src siRNA treatment (Supplementary Fig. S7e,f). There were no significant changes in apoptosis between any groups (data not shown). As our data indicate that increased adrenergic signalling results in increased invasion in vitro, we analysed $\mathrm{H} \& \mathrm{E}$ sections obtained from tumour bearing mice undergoing restraint stress. These results show that restraint stress leads to tumour infiltration into underlying tissue and Src siRNA-DOPC abrogates this effect (Supplementary Fig. S7g). Moreover, we found that tumours from mice undergoing restraint stress had elevated $\beta$-catenin levels, while Src siRNA-DOPC blocks this increase (Supplementary Fig. S7h).

To determine the contribution of ADRB in the daily restraint model, we treated control or restrained mice ( $n=10$ per group) bearing HeyA8 tumours with propranolol. As expected, propranolol abrogated the daily restraint-induced increase in tumour growth (Fig. 5e). Tumours from animals treated with propranolol and exposed to daily restraint stress had substantially decreased levels of $\mathrm{pSrc}^{\mathrm{Y} 419}$ compared with mice exposed to daily 
a

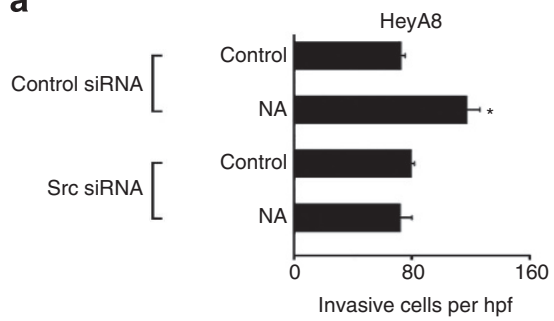

C

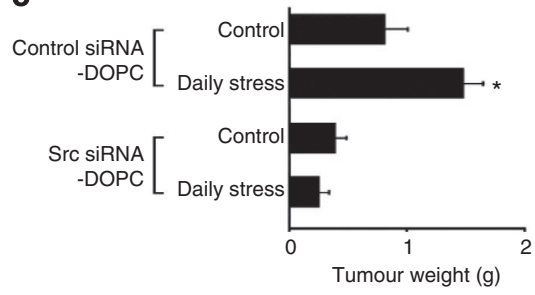

e

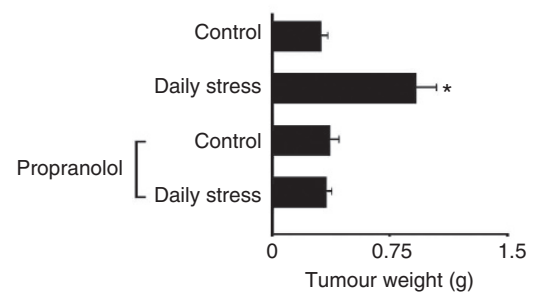

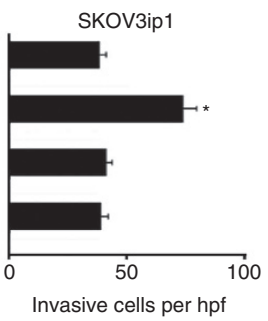

b

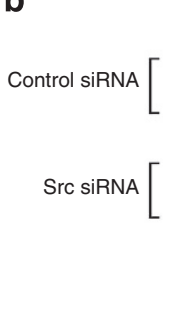

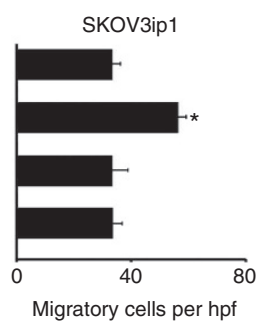

d

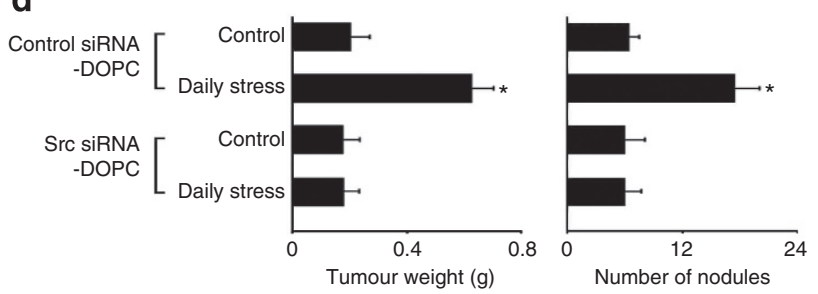

f
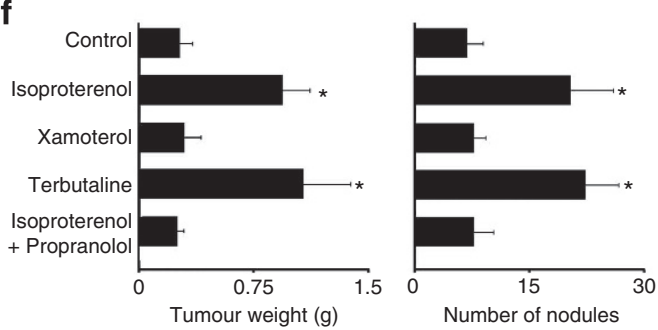

Figure 5 | Adrenergic-mediated Src activation leads to increased tumour invasion and growth. NA $10 \mu M$ induces HeyA8 and SKOV3ip1 (a) cell invasion and (b) migration. (c) Mice were inoculated with either HeyA8 $\left(2.5 \times 10^{5}\right)$ or $(\mathbf{d})$ SKOV3ip1 $\left(1.0 \times 10^{6}\right)$ cells and subjected to daily restraint and treated twice a week with either control siRNA-DOPC or Src siRNA-DOPC. Treatment with Src siRNA-DOPC blocked the daily restraint-mediated induction in tumour weight and number of nodules compared with control siRNA-DOPC. (e) Mice bearing SKOV3ip1 tumours undergoing daily restraint were treated daily with propranolol $\left(2 \mathrm{mg} \mathrm{kg}^{-1}\right)$. Propranolol counteracts the effects of daily restraint on tumour growth. (f) Mice bearing SKOV3ip1 tumours were treated daily with either: $10 \mathrm{mg} \mathrm{kg}^{-1}$ isoproterenol, $5 \mathrm{mg} \mathrm{kg}^{-1}$ terbutaline, $1 \mathrm{mg} \mathrm{kg}^{-1}$ xamoterol or isoproterenol plus $2 \mathrm{mg} \mathrm{kg}^{-1}$ of propranolol.

Isoproterenol and terbutaline induced tumour growth, but not xamoterol. Mice treated with isoproterenol plus propranolol have tumour burden similar to control mice.

restraint stress (Supplementary Fig. S7i). To further delineate the role of ADRB on tumour growth, we treated HeyA8 tumour bearing mice ( $n=10$ mice) with either isoproterenol, xamoterol, terbutaline or isoproterenol plus propranolol. As expected, isoproterenol significantly increased tumour growth, and a similar increase in tumour burden was noted with terbutaline (Fig. 5f). However, treatment with xamoterol or propranolol in combination with isoproterenol did not result in increased tumour growth compared with the control group (Fig. 5f). Tumours from animals exposed to isoproterenol or terbutaline had substantially increased levels of $\mathrm{pSrc}^{\mathrm{Y} 419}$ and $\mathrm{pSrc}^{\mathrm{S} 17}$, while xamoterol or propranolol in combination with isoproterenol did not induce phosphorylation at these sites (Supplementary Fig. S7j). Additionally, bioluminescence imaging analysis revealed that daily restraint stress resulted in significantly increased tumour growth and metastasis, which was abrogated by the use of propranolol (Supplementary Fig. S7k). Next, to determine the effects of daily restraint stress on the patterns of metastasis, we utilized a fully orthotopic mouse. SKOV3ip1 ovarian cancer cells were injected directly into the right ovary of nude mice followed by exposure to daily restraint stress, with or without Src siRNADOPC treatment. Daily restraint stress resulted in significantly higher tumour nodule counts and distant metastatic spread compared with control siRNA-DOPC (Supplementary Fig. S7l). Src siRNA-DOPC completely abrogated the effects of stress on tumour metastasis (Supplementary Fig. S7l). To further delineate the role of ADRB2 in vivo, we inoculated mice ( $n=7$ per group) with A2780-OG2 (empty vector), A2780-ADRB2 or A2780 cells into the subcutaneous space and treated groups with isoproterenol or PBS. Isoproterenol significantly increased tumour growth in the A2780-ADRB2 group compared with the A2780-OG2 group, whereas the A2780 group did not respond to isoproterenol treatment (Supplementary Fig. S7m). To examine the role of increased peripheral nervous system activity on tumour growth, we performed an experiment where mice undergoing daily restraint stress were inoculated with SKOV3ip1 cells and treated with the peripheral ganglionic blocker hexamethonium bromide (daily dose of $1 \mathrm{mg} \mathrm{kg}^{-1}$ ). This treatment completely blocked the daily restraint-induced tumour growth (data not shown). Next, we used ID8 ${ }^{\mathrm{VEGF}}$ murine ovarian cancer cells transfected with human WT Src or S17A mutated Src. These cells were then injected subcutaneously into the right flank of C57 mice, treated with murine Src siRNADOPC (to silence endogenous Src; Supplementary Fig. S7n) and isoproterenol. Isoproterenol treatment resulted in significantly increased tumour growth in mice inoculated with WT Src-cells, but not with S17A Src-cells (Supplementary Fig. S7n).

pSrc $^{\mathrm{Y} 419}$ expression in human ovarian carcinoma. To determine whether adrenergic activity might relate to Src activation in human cancers, we examined 91 invasive epithelial ovarian 
a
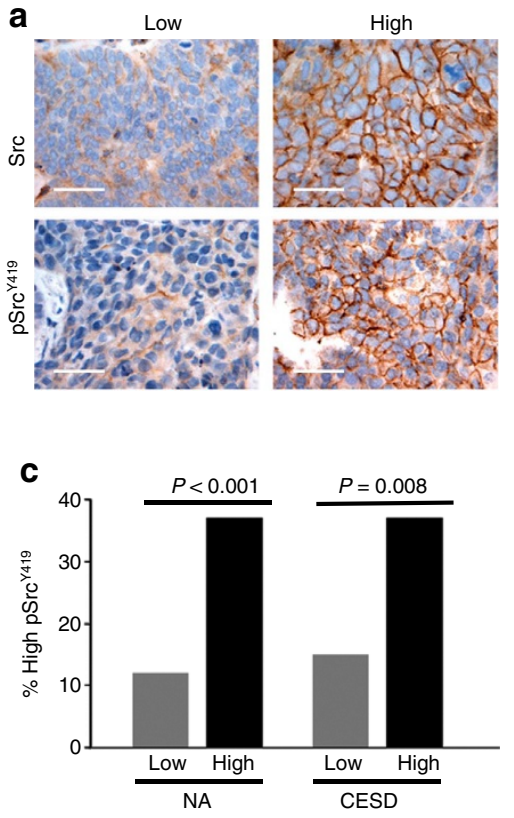

b

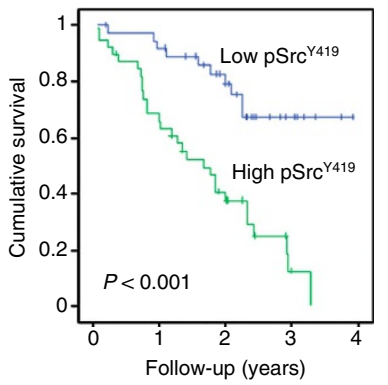

e

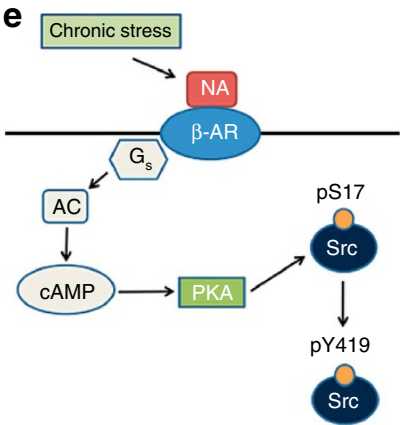

d

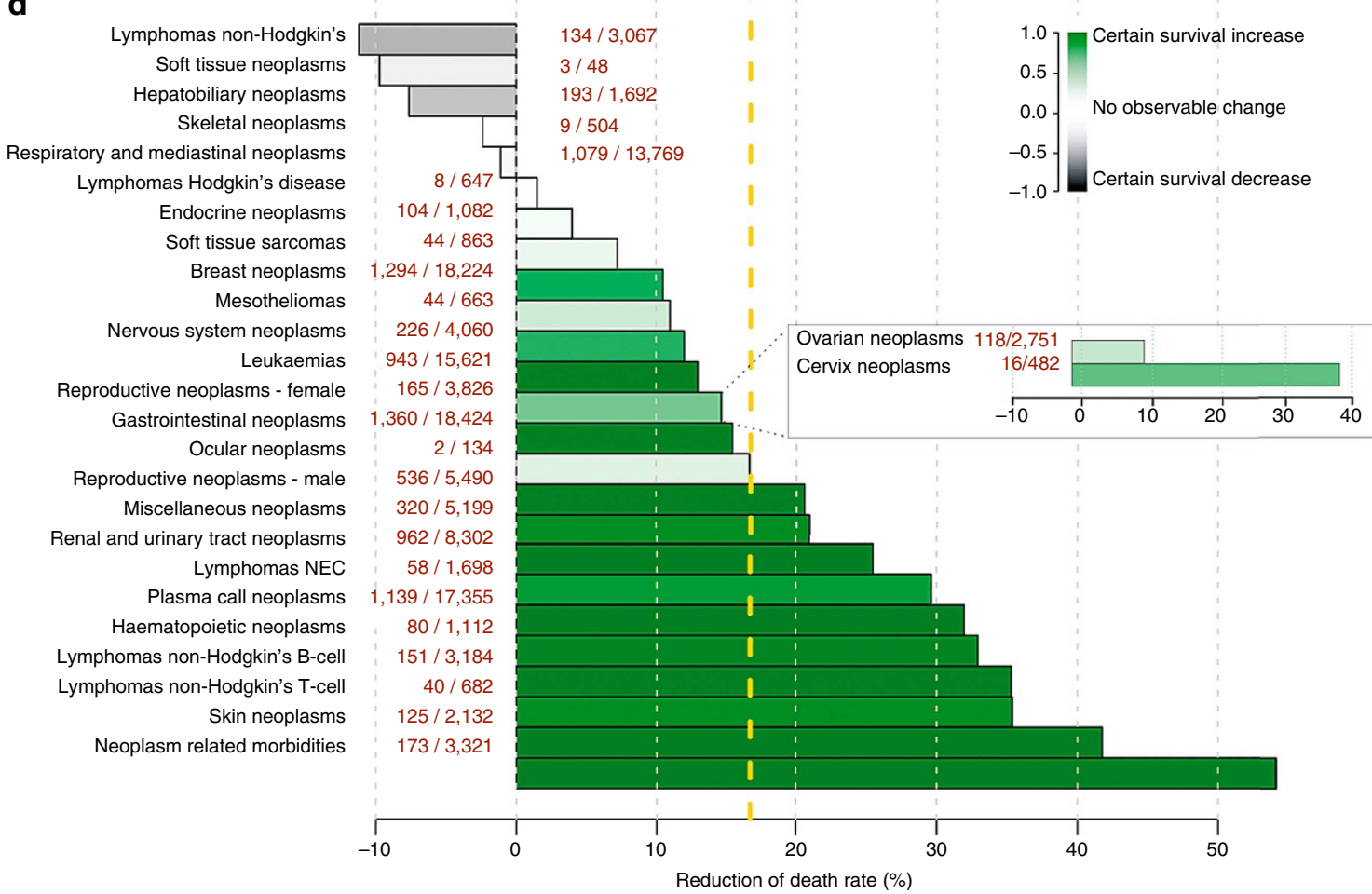

Figure 6 | High pSrc ${ }^{Y 419}$ levels are associated with decreased survival and depressive symptoms. (a) Representative images of human ovarian tumours with low or high expression of Src and pSrc ${ }^{\mathrm{4} 19}$. Images were taken at original magnification $\mathrm{X} 200$ (scale bar $=50 \mu \mathrm{m}$ ). (b) Kaplan-Meier curves of disease-specific mortality for patients with epithelial ovarian carcinoma $(n=91)$ based on $\mathrm{pSrc}{ }^{\Upsilon 419}$ expression. The log-rank test (two-sided) was used to compare differences between groups. (c) Percentage of ovarian cancers with high pSrc ${ }^{\mathrm{Y} 419}$ expression based on tumoural NA levels (greater than the median value of $0.84 \mathrm{pg} \mathrm{mg}^{-1}$ versus less than $0.84 \mathrm{pg} \mathrm{mg}^{-1}$ ) and CESD scores $\geq 16$. (d) Effect of beta blocker usage on cancer-related mortality, as estimated based on data from the FDA Adverse Event Reporting System. Bars, mortality decrease (green) or increase (black) by cancer type; saturation representing statistical confidence. Dashed yellow line, general mortality reduction over all cancer-related cases (17\%). Numbers, cases having received a beta-blocker/total number of cases with given cancer type. (e) In response to chronic stress, catecholamines are released from the sympathetic nervous system. Stress-related hormones bind and activate ADRB receptors on tumour cells, initiating a cascade of events that result in Src activation.

cancers. Consistent with prior reports ${ }^{23,24}$, increased Src expression was noted in $88 \%$ of the tumour samples, while elevated $\mathrm{pSrc}^{\mathrm{Y} 419}$ expression was noted in $42 \%$ (Fig. 6a). We found that elevated levels of $\mathrm{pSrc}^{\mathrm{Y} 419}$ were associated with poor mean patient survival by univariate analysis ( 1.67 years versus not yet reached; $P<0.001$; Fig. $6 \mathrm{~b})$. As depression, as measured by 
high scores on the Centre for Epidemiological Studies Depression scale (CES-D $)^{25,26}$, has been linked to increased tumour catecholamine levels ${ }^{27,28}$, we examined potential relationships between CESD scores and Src activity. Patients with high CES-D scores $(\geq 16)$ had significantly higher levels of tumoural $\mathrm{pSrc}^{\mathrm{Y} 419}$ $(P=0.008)$ compared with those with low scores (Fig. 6c). In addition, NA levels above the median were significantly associated with increased $\mathrm{pSrc}^{\mathrm{Y} 419}$ expression $(P<0.001)$, but not with increases in total Src (Fig. 6c). In a subset of these tumour samples, $\mathrm{pSrc}^{\mathrm{Y} 419}$ and $\mathrm{pSrc}^{\mathrm{S} 17}$ levels were evaluated by ELISA and western blot analyses, respectively. There was a significant association between elevated $\mathrm{pSrc}^{\mathrm{Y} 419}$ and $\mathrm{pSrc}^{\mathrm{S} 17}$ in these samples (Supplementary Fig. S8a). Moreover, we found a strong positive correlation between tumoural NA and $\mathrm{pSrc}^{\mathrm{Y} 419}$ and $\mathrm{pSrc}^{\mathrm{S} 17}$ levels (Supplementary Fig. S8b,c).

Beta blockers may reduce cancer-related mortality. To examine the potential clinical impact of our findings, we asked whether chemical perturbation of beta-adrenergic function in cancer patients might result in lower patient mortality. To test this hypothesis, we employed adverse events data from the FDA's Adverse Event Reporting System (AERS; http://www.fda.gov/ Drugs/GuidanceComplianceRegulatoryInformation/Surveillance) to examine whether usage of beta blockers by patients affected cancer-related mortality. Our analysis revealed that mortality (that is, 'Death' reported either as a patient's outcome or as a patient' reaction), was reduced by an average of $17 \%$ across all major cancer types if patients were treated with beta blockers (Fig. 6d). Moreover, a $14.64 \%$ decrease in mortality was observed among patients with ovarian and cervical cancer. These data suggest that beta blocker use among cancer patients can significantly reduce cancer-related mortality.

\section{Discussion}

Here, we describe a unique mechanism by which increased adrenergic signalling results in Src activation, which induces downstream proteins important for cell survival, motility and invasion ${ }^{29-31}$. Increased serine phosphorylation at Src amino terminus following cAMP treatment was demonstrated 30 years $\mathrm{ago}^{32}$, and a consensus PKA site at $\mathrm{Src}^{\mathrm{S1}}$ was subsequently identified ${ }^{33}$. However, no physiological role for phosphorylation at $\mathrm{Src}^{\mathrm{S} 17}$ had been established; $\mathrm{pSrc}^{\mathrm{S} 17}$ can mediate Rap1 activation and inhibit ERK by cAMP-dependent pathways ${ }^{34,35}$. Src has been implicated in NA-stimulated VEGF production by adipocytes $^{36,37}$, and in NA-stimulated IL-6 production by cancer cells $^{7}$. Although different mechanisms have been suggested to account for ADRB-mediated Src activation (for example, $\beta$-arrestin and EGFR-dependent Src phosphorylation ${ }^{38}$ ), the precise mechanisms mediating ADRB/cAMP/PKA-induced Src activation or the resultant biological effects were not well understood. Our results have identified a new functional role for $\mathrm{Src}^{\mathrm{S} 17}$ as a key molecular switch that links a serine kinase to downstream tyrosine kinase signalling and disease progression (Fig. 6e). Specifically, our results indicate that the neuroendocrine stress response can directly affect tumour growth and malignant progression through receptors expressed on tumour cells that lead to a critical phosphorylation event, resulting in Src activation. NA is the most abundant stress hormone in the ovary 39,40 and its levels are much higher in the ovary than in the plasma ${ }^{41,42}$. To the extent that biobehavioral states can modulate catecholamine levels in the tumour microenvironment, these findings offer new opportunities for designing interventions to protect individuals from the harmful effects of chronic adrenergic stimulation ${ }^{43}$.

A number of studies have recently emerged supporting the rationale for designing clinical studies to target neuroendocrine function, which could represent a new avenue for treating individuals with cancer ${ }^{44}$. On the basis of our work, betaantagonists can abrogate many of the deleterious effects of increased adrenergic signalling. For example, among prostate cancer patients taking anti-hypertensive medication, only beta blockers were associated with a reduction of cancer risk ${ }^{44}$, while others have shown a reduction in overall cancer risk ${ }^{45}$. Moreover, our findings support the use of Src family kinase inhibitors as tools to block the deleterious effects of increased sympathetic activity $^{46,47}$. Collectively, our data represent a new understanding of Src regulation in response to adrenergic signalling in cancer cells and provide a biologically plausible and potent way of inhibiting tumour progression among cancer patients.

\section{Methods}

Proteomic analysis. Two-dimensional gel electrophoresis was conducted as first described by O'Farrell ${ }^{48}$. All biological samples were run in duplicate (technical). After electrophoresis, the gels were fixed and either directly stained with SYPRORube (Bio-Rad, Hercules, CA) or sequentially stained with ProQ-Diamond (detects phosphate groups attached to tyrosine, serine or threonine residues) and SYPRORuby (detects total protein). Gels were then scanned at a $100-\mathrm{mm}$ resolution using the Perkin-Elmer ProEXPRESS 2D Proteomic Imaging System (Boston, MA). After quantifying the relative spot intensities among samples and normalizing the phosphorylation levels to the total amount of protein, gel spots were excised and prepared for MALDI-TOF-MS analysis using DigiLab's (Holliston, MO) ProPic and ProPrep robotic instruments following the manufacturer's protocol. MALDITOF/TOF was performed using the Applied Biosystems 4800 MALDI TOF/TOF analyser for peptide mass fingerprinting and sequencing (See Supplementary Procedures for a more detailed description). Protein identification was performed using a Bayesian algorithm, where high probability matches are indicated by an expectation score, which is an estimate of the number of matches that would be expected in that database if the matches were completely random ${ }^{49}$. See Supplementary Procedures for expanded methodology.

Signalling network analysis. We sought to analyse whether a key mediator kinase might exist that is capable of directly or indirectly explaining the majority of the observed differences in phosphorylation and protein abundance. We restricted the length of signalling chains to include at most two phosphorylation events (equivalently, allowing for at most one intermediate kinase, because otherwise, the set of potential candidates would suffer from 'combinatorial explosion'). We constructed this two-layer phosphorylation network upstream of the identified proteins using information from Phospho.ELM and networKIN ${ }^{50,51}$. In the resultant network, 45 of the dysregulated proteins could be linked to at least one of 243 kinases. Scoring and sorting these candidates by the number of downstream dysregulated proteins suggested Src as a tentative key mediator for the experimentally observed differences (37/45 proteins, Fig. 1 and Supplementary Fig. S2a). We note that this neither disproves an important role for other kinases nor proves that Src is the most important kinase in this context; however, it does illustrate some potential for Src being of central importance in the cellular response to NA.

Western blot analysis. Cell lysates were prepared by washing cells with phosphate-buffered saline and incubating them for $10 \mathrm{~min}$ at $4{ }^{\circ} \mathrm{C}$ in modified radioimmunoprecipitation assay lysis buffer. Cells were scraped from plates and centrifuged for $20 \mathrm{~min}$ at $4{ }^{\circ} \mathrm{C}$, and the supernatant was collected. Protein concentrations were determined using a BCA reagent kit (Pierce), and $40 \mu \mathrm{g}$ of wholecell lysates were separated by $10 \%$ SDS-PAGE. Samples were transferred to a nitrocellulose membrane by wet electrophoresis (Bio-Rad), blocked with 5\% nonfat milk for $1 \mathrm{~h}$ at room temperature, and incubated with $\mathrm{Src}, \mathrm{pSrc}^{\mathrm{Y} 416}, \mathrm{pSrc}^{\mathrm{S} 17}$ or pSrc ${ }^{\mathrm{Y} 530}$ overnight at $4{ }^{\circ} \mathrm{C}$. Primary antibody was detected with anti-rabbit IgG (Amersham Biosciences, Piscataway, NJ) and developed with an enhanced chemiluminescence detection kit (Pierce, Rockford, IL).

siRNA preparation and treatment. We used siRNA to downregulate Src in vitro and in vivo. Src-specific siRNA was purchased from Ambion (Austin, TX) with the following target sequence: $5^{\prime}$-GGCTGAGGAGTGGTATTTT-3'. Additional validated Src sequences were purchased from Sigma-Genosys (The Woodlands, TX). Control siRNA used was obtained from Qiagen (Valencia, CA) with the following target sequence: $5^{\prime}$-AATTCTCCGAACGTGTCACGT-3'. For in vitro studies, cells were transfected with siRNA that was incorporated into Lipofectamine 2000 transfection reagent (Invitrogen, Carlsbad, CA) following the manufacturer's protocol. After $72 \mathrm{~h}$, the experiments were carried out. For in vivo studies, siRNA was incorporated into the neutral liposome DOPC as previously described ${ }^{52,53}$. For each treatment, $3.5 \mu \mathrm{g}$ of siRNA was reconstituted in $200 \mu \mathrm{l}$ of phosphatebuffered saline and administered by intravenous injection. The dosing schedule is described in the in vivo tumour model below. 
Migration and invasion assay. The membrane invasion culture system chamber was used to measure the in vitro invasive and migratory potential of all cell lines used in this study. When required, siRNA was added to cells $24 \mathrm{~h}$ before cell harvest, and NA was added at the start of the experiment. The membrane invasion culture system assay was performed as previously described. Briefly, single cell tumour suspensions were seeded into the upper wells at a concentration of $1 \times 10^{5}$ cells per well for the invasion assays and $7.5 \times 10^{4}$ cells per well for the migration assays. For the invasion and migration assays, a human basement membrane and gelatin-coated membrane were used, respectively. Cells were allowed to invade for $24 \mathrm{~h}$ or migrate for $6 \mathrm{~h}$ for the invasion and migration assays, respectively. The cells were then collected, fixed, stained and counted by light microscopy.

Chronic stress model. We obtained 8- to 12-week-old female athymic nude mice from the National Cancer Institute. All experiments were approved by the Institutional Animal Care and Use Committee at MD Anderson Cancer Center. To experimentally induce stress in the mice, we used a restraint-stress procedure that utilizes a physical restraint system that we developed. Tumour cells were injected intraperitoneally or subcutaneously into mice in all groups 7 days after the stress procedure began. A total of 10 mice per group were used. Starting 4 days after tumour cell injection, mice were treated with siRNA (control or Src-specific, $3.5 \mu \mathrm{g}$ in DOPC every 3 days, intraperitoneally) for the duration of the experiments. We necropsied the animals 21 days after tumour cell injection. At this time, the entire peritoneal cavity was examined for identifiable disease, and mouse weight, tumour weight and the distribution of tumour was recorded by gynaecological oncologists.

\section{Centre for epidemiological studies depression scale. Patients completed} psychosocial questionnaires between their initial preoperative appointment and surgery. The CES-D scale is a 20 -item measure that assesses depressive symptomatology over the last week ${ }^{25}$. Scores of 16 or higher have been associated with clinical depression.

Statistical analysis. We compared continuous variables using either Student's $t$-test or analysis of variance and compared the categorical variables using the $\chi^{2}$ test. We used a nonparametric test (Mann-Whitney test), when appropriate, to compare differences. We considered $P<0.05$ to be significant.

Additional methods. Details of reagents, cell culture conditions, mass spectrometry, protein identification analysis, microarray analysis, plasmids, kinase assay, immunochemistry, immunoprecipitation and methods used to determine NA concentration, molecular dynamic simulations and the bioinformatics analysis of the FDA adverse event reports can be found in the Supplementary Methods.

\section{References}

1. Antoni, M. H. et al. The influence of bio-behavioural factors on tumour biology: pathways and mechanisms. Nat. Rev. Cancer 6, 240-248 (2006).

2. Thaker, P. H. et al. Chronic stress promotes tumor growth and angiogenesis in a mouse model of ovarian carcinoma. Nat. Med. 12, 939-944 (2006).

3. Sood, A. K. et al. Adrenergic modulation of focal adhesion kinase protects human ovarian cancer cells from anoikis. J. Clin. Invest. 120, 1515-1523 (2010).

4. Sood, A. K. et al. Stress hormone-mediated invasion of ovarian cancer cells. Clin. Cancer Res. 12, 369-375 (2006).

5. Shahzad, M. M. et al. Stress effects on FosB- and interleukin-8 (IL8)-driven ovarian cancer growth and metastasis. J. Biol. Chem. 285, 35462-35470 (2010).

6. Landen, Jr C. N. et al. Neuroendocrine modulation of signal transducer and activator of transcription-3 in ovarian cancer. Cancer Res. 67, 10389-10396 (2007).

7. Nilsson, M. B. et al. Stress hormones regulate interleukin-6 expression by human ovarian carcinoma cells through a Src-dependent mechanism. J. Biol. Chem. 282, 29919-29926 (2007).

8. Peleg, D., Arbogast, L. A., Peleg, E. \& Ben-Jonathan, N. Predominance of L-dopa in fetal plasma and the amniotic fluid during late gestation in the rat. Am. J. Obstet. Gynecol. 149, 880-883 (1984).

9. Lutgendorf, S. K. et al. Stress-related mediators stimulate vascular endothelial growth factor secretion by two ovarian cancer cell lines. Clin. Cancer Res. 9, 4514-4521 (2003).

10. Thomas, S. M. \& Brugge, J. S. Cellular functions regulated by Src family kinases. Annu. Rev. Cell Dev. Biol. 13, 513-609 (1997).

11. Xu, W., Harrison, S. C. \& Eck, M. J. Three-dimensional structure of the tyrosine kinase c-Src. Nature 385, 595-602 (1997).

12. Xu, W., Doshi, A., Lei, M., Eck, M. J. \& Harrison, S. C. Crystal structures of c-Src reveal features of its autoinhibitory mechanism. Mol. Cell. 3, 629-638 (1999).

13. Trott, O. \& Olson, A. J. AutoDock Vina: improving the speed and accuracy of docking with a new scoring function, efficient optimization, and multithreading. J. Comput. Chem. 31, 455-461 (2010).
14. Dundas, J. et al. CASTp: computed atlas of surface topography of proteins with structural and topographical mapping of functionally annotated residues. Nucleic Acids Res. 34, W116-W118 (2006).

15. Roberts, E., Eargle, J., Wright, D. \& Luthey-Schulten, Z. MultiSeq: unifying sequence and structure data for evolutionary analysis. BMC Bioinformatics 7, 382 (2006).

16. Humphrey, W., Dalke, A. \& Schulten, K. VMD: visual molecular dynamics. $J$ Mol. Graph. 14, 33-38 27-38 (1996).

17. Nagar, B. et al. Structural basis for the autoinhibition of c-Abl tyrosine kinase. Cell 112, 859-871 (2003).

18. Young, T., Abel, R., Kim, B., Berne, B. J. \& Friesner, R. A. Motifs for molecular recognition exploiting hydrophobic enclosure in protein-ligand binding. Proc. Natl Acad. Sci. USA 104, 808-813 (2007).

19. Lazaridis, T. Inhomogeneous fluid approach to solvation thermodynamics. J. Phys. Chem. B 102, 3531-3541 (2011).

20. Li, Z. \& Lazaridis, T. Computing the thermodynamic contributions of interfacial water. Methods Mol. Biol. 819, 393-404 (2012).

21. Baker, N. A., Sept, D., Joseph, S., Holst, M. J. \& McCammon, J. A. Electrostatics of nanosystems: application to microtubules and the ribosome. Proc. Natl Acad. Sci USA 98, 10037-10041 (2001).

22. Simons, K. T., Bonneau, R., Ruczinski, I. \& Baker, D. Ab initio protein structure prediction of CASP III targets using ROSETTA. Proteins Suppl 3, 171-176 (1999).

23. Wiener, J. R. et al. Activated SRC protein tyrosine kinase is overexpressed in late-stage human ovarian cancers. Gynecol. Oncol. 88, 73-79 (2003).

24. Budde, R. J., Ke, S. \& Levin, V. A. Activity of pp60c-src in 60 different cell lines derived from human tumors. Cancer Biochem. Biophys. 14, 171-175 (1994).

25. Radloff, L. S. The CES-D scale. Social support, life events, and depression. Appl. Psychol. Meas. 1, 385-401 (1977).

26. Ensel, W. M. Measuring Depression: the CES-D Scale. Social Support, Life Events, and Depression (Academic Press, 1986).

27. Lutgendorf, S. K. et al. Depression, social support, and beta-adrenergic transcription control in human ovarian cancer. Brain Behav. Immun. 23, 176-183 (2009).

28. Hughes, J. W., Watkins, L., Blumenthal, J. A., Kuhn, C. \& Sherwood, A. Depression and anxiety symptoms are related to increased 24-hour urinary noradrenaline excretion among healthy middle-aged women. J. Psychosom. Res 57, 353-358 (2004).

29. Kim, M. J. et al. Downregulation of FUSE-binding protein and c-myc by tRNA synthetase cofactor p38 is required for lung cell differentiation. Nat. Genet. 34, 330-336 (2003).

30. Obermeier, A. et al. PAK promotes morphological changes by acting upstream of Rac. EMBO J. 17, 4328-4339 (1998).

31. Meylan, E., Martinon, F., Thome, M., Gschwendt, M. \& Tschopp, J. RIP4 (DIK/ PKK), a novel member of the RIP kinase family, activates NF-kappa B and is processed during apoptosis. EMBO Rep. 3, 1201-1208 (2002).

32. Cross, F. R. \& Hanafusa, H. Local mutagenesis of Rous sarcoma virus: the major sites of tyrosine and serine phosphorylation of pp60src are dispensable for transformation. Cell 34, 597-607 (1983).

33. Brown, M. T. \& Cooper, J. A. Regulation, substrates and functions of src. Biochim. Biophys. Acta 1287, 121-149 (1996).

34. Obara, Y., Labudda, K., Dillon, T. J. \& Stork, P. J. PKA phosphorylation of Src mediates Rap1 activation in NGF and cAMP signaling in PC12 cells. J. Cell Sci. 117, 6085-6094 (2004).

35. Schmitt, J. M. \& Stork, P. J. PKA phosphorylation of Src mediates cAMP's inhibition of cell growth via Rap1. Mol. Cell 9, 85-94 (2002).

36. Fredriksson, J. M. \& Nedergaard, J. Noradrenaline specifically stimulates ribonucleotide reductase subunit R2 gene expression in proliferating brown adipocytes: mediation via a cAMP/PKA pathway involving Src and Erk1/2 kinases. Exp. Cell Res. 274, 207-215 (2002).

37. Fredriksson, J. M., Lindquist, J. M., Bronnikov, G. E. \& Nedergaard, J. Noradrenaline induces vascular endothelial growth factor gene expression in brown adipocytes through a beta -adrenoreceptor/cAMP/protein kinase A pathway involving Src but independently of Erk1/2. J. Biol. Chem. 275, 13802-13811 (2000).

38. Gschwind, A., Zwick, E., Prenzel, N., Leserer, M. \& Ullrich, A. Cell communication networks: epidermal growth factor receptor transactivation as the paradigm for interreceptor signal transmission. Oncogene 20, 1594-1600 (2001).

39. Aguado, L. I. \& Ojeda, S. R. Prepubertal ovarian function is finely regulated by direct adrenergic influences. Role of noradrenergic innervation. Endocrinology 114, 1845-1853 (1984).

40. Ben-Jonathan, N., Arbogast, L. A., Rhoades, T. A. \& Bahr, J. M. Noradrenaline in the rat ovary: ontogeny and de novo synthesis. Endocrinology 115, 1426-1431 (1984).

41. Lara, H. E. et al. Changes in sympathetic nerve activity of the mammalian ovary during a normal estrous cycle and in polycystic ovary syndrome: Studies on noradrenaline release. Microsc. Res. Tech. 59, 495-502 (2002). 
42. Schmidt, C. \& Kraft, K. Beta-endorphin and catecholamine concentrations during chronic and acute stress in intensive care patients. Eur. J. Med. Res. 1, 528-532 (1996).

43. Armaiz-Pena, G. N., Cole, S. W., Lutgendorf, S. K. \& Sood, A. K. Neuroendocrine influences on cancer progression. Brain Behav. Immun. DOI: 10.1016/j.bbi.2012.06.005 (2012).

44. Perron, L., Bairati, I., Harel, F. \& Meyer, F. Antihypertensive drug use and the risk of prostate cancer (Canada). Cancer Cause Control 15, 535-541 (2004).

45. Algazi, M., Plu-Bureau, G., Flahault, A., Dondon, M. G. \& Le, M. G. [Could treatments with beta-blockers be associated with a reduction in cancer risk?]. Revue d'epidemiologie et de Sante Publique 52, 53-65 (2004).

46. Kopetz, S., Shah, A. N. \& Gallick, G. E. Src continues aging: current and future clinical directions. Clin. Cancer Res. 13, 7232-7236 (2007).

47. Nam, S. et al. Action of the Src family kinase inhibitor, dasatinib (BMS354825), on human prostate cancer cells. Cancer Res. 65, 9185-9189 (2005).

48. O'Farrell, P. H. High resolution two-dimensional electrophoresis of proteins J. Biol. Chem. 250, 4007-4021 (1975).

49. Ryckaert, J.-P., Ciccotti, G. \& Berendsen, H. J. C. Numerical integration of the cartesian equations of motion of a system with constrains: molecular dynamics of n-alkenes. J. Comput. Phys. 23, 327-341 (1977).

50. Diella, F. et al. Phospho.ELM: a database of experimentally verified phosphorylation sites in eukaryotic proteins. BMC Bioinformatics. 5, 79, (2004).

51. Linding, R. et al. Systematic discovery of in vivo phosphorylation networks. Cell 129, 1415-1426 (2007).

52. Landen, Jr C. N. et al. Therapeutic EphA2 gene targeting in vivo using neutral liposomal small interfering RNA delivery. Cancer Res. 65, 6910-6918 (2005).

53. Pecot, C. V., Calin, G. A., Coleman, R. L., Lopez-Berestein, G. \& Sood, A. K RNA interference in the clinic: challenges and future directions. Nat. Rev. Cancer 11, 59-67 (2011).

\section{Acknowledgements}

GNA-P was supported, in part, by the NCI F31CA126474 Fellowship for Minority Students. Y.G.L., L.Y.H., A.M.N. and R.L.S. were supported by an NIH T32 Training Grant CA101642. S.K.L. was supported by NIH grants CA140933 and CA104825. M.M.S. was supported by the GCF-Molly Cade ovarian cancer research grant and the NIH/ NICHD Baylor WRHR scholarship grant. This work was also supported in part by NIH grants (CA110793 and CA109298, P50CA083639, P50CA098258, CA128797,
RC2GM092599, U54CA151668, U54CA96300 and U54CA96297), the University of Texas Medical Branch NHLBI Proteomics Centre (contract HV-10-05_(2)), Ovarian Cancer Research Fund Programme Project Development Grant, Department of Defence (OC073399, W81XWH-10-1-0158, OC100237 and BC085265), the Zarrow Foundation, the Betty Ann Asche Murray Distinguished Professorship, the Marcus Foundation, the RGK Foundation, the Gilder Foundation, the estate of C.G. Johnson Jr, the Laura and John Arnold Foundation) and the Blanton-Davis Ovarian Cancer Research Programme. The authors thank Ms Donna Reynolds and Drs Robert Langley, and Philip Stork for helpful discussions, comments and for providing reagents. We would like to thank the High Performance Computing Facility of the University of Puerto Rico for the computer time.

\section{Author contributions}

G.N.A.-P. designed the studies, performed experiments and wrote the manuscript. A.C., M.T.-L., T.Y. and G.E.L. designed and performed structural modelling analyses and edited the manuscript. Z.W., R.D.E., K.V.S. and J.E.W. performed 2D gel analyses and mass spectrometry studies and edited the manuscript. D.B.J., T.G.S. and A.Z. analysed the proteomics data and performed the adverse event data set bioinformatics analysis. Y.G.L., L.Y.H., A.M.N., R.L.S., L.S.M., J.K.A., A.S.N., K.M.G. and M.M.K.S. performed experiments and edited the manuscript. P.V.-M, C.R.-A. and G.L.-B. performed siRNA incorporations and edited the manuscript. G.J.V., M.Z. and M.B.-E. designed and performed plasmid transfections and edited the manuscript. M.T.D. performed the immunohistochemical analysis and edited the manuscript. K.D.G., G.E.G., S.W.C., S.K.L. and A.K.S. designed the overall study, analysed data and edited the manuscript.

\section{Additional information}

Supplementary Information accompanies this paper at http://www.nature.com/ naturecommunications

Competing financial interests: The authors declare no competing financial interests.

Reprints and permission information is available online at http://npg.nature.com/ reprintsandpermissions/

How to cite this article: Armaiz-Pena, G. N. et al. Src activation by $\beta$-adrenoreceptors is a key switch for tumor metastasis. Nat. Commun. 4:1403 doi: 10.1038/ncomms2413 (2013). 


\section{Corrigendum: Src activation by $\beta$-adrenoreceptors is a key switch for tumour metastasis}

Guillermo N. Armaiz-Pena, Julie K. Allen, Anthony Cruz, Rebecca L. Stone, Alpa M. Nick, Yvonne G. Lin, Liz Y. Han, Lingegowda S. Mangala, Gabriel J. Villares, Pablo Vivas-Mejia, Cristian Rodriguez-Aguayo, Archana S. Nagaraja, Kshipra M. Gharpure, Zheng Wu, Robert D. English, Kizhake V. Soman, Mian M.K. Shahzad, Maya Zigler, Michael T. Deavers, Alexander Zien, Theodoros G. Soldatos, David B. Jackson, John E. Wiktorowicz, Madeline Torres-Lugo, Tom Young, Koen De Geest, Gary E. Gallick, Menashe Bar-Eli, Gabriel Lopez-Berestein, Steve W. Cole, Gustavo E. Lopez, Susan K. Lutgendorf \& Anil K. Sood

Nature Communications 4:1403 doi: 10.1038/ncomms2413 (2013); Published 29 Jan 2013; Updated 29 Jul 2013

The original version of this Article contained a typographical error in the spelling of the author Mian M.K. Shahzad, which was incorrectly given as Mian M.K. Shazhad. This has now been corrected in both the PDF and HTML versions of the Article. 